\title{
The Weakest Point Evaluation Method for the Control Range of the Sound Velocity Profile Station
}

\author{
Liu Guoqing, Jin Shaohua, and You Baoping \\ Department of Military Oceanography and Hydrography and Cartography, Dalian Naval Academy, Dalian 116018, China
}

\begin{abstract}
Sound velocity measurement is an important part of multi-beam sounding, and its accuracy directly affects the coordinate reduction of sounding points. Because of the variability of seawater sound velocity in time and space, the layout density of acoustic section stations directly affects the accuracy of multi-beam sounding. Over-dense layout wastes resources and causes inefficiency; over-sparse layout is not accurate enough to control the entire survey area. Based on the constant gradient acoustic ray tracing algorithm, this paper constructs the accuracy evaluation model of the weakest water depth point of acoustic profile station control, and analyses whether the existing multi-beam acoustic profile station layout scheme can satisfy the accuracy requirements of multi-beam sounding through an example calculation, which provides a theoretical basis for adjusting the layout scheme of acoustic profile station for field surveyors.
\end{abstract}

\section{Introduction}

Sound velocity distribution is a very important parameter in the field of underwater acoustics in the marine environment ${ }^{[1]}$. Because seawater is a highly mobile medium, its temperature, salinity and density characteristics are not only affected by the invasion of runoff freshwater and high salinity of ocean currents, but also by temperature, season, flow field and other factors. Significant changes in temperature and Salinity Characteristics of seawater media will inevitably lead to spatial and temporal changes in sound velocity structure, which will have a significant impact on multi-beam seabed exploration [2]. Multi-beam measurement is strongly dependent on sound velocity structure. In order to improve the accuracy of multi-beam measurement, it is necessary to control the measurement error of sound velocity profile ${ }^{[3]}$. In the course of multi-beam measurement, because of the variability of sound velocity profile in time and space, in principle, the more and more sound velocity profile stations, the more accurate they are. But in practice, the inefficient measurement scheme of infinite sound velocity profile stations can not be adopted. In order to satisfy the accuracy, and taking into account the variability of time and space of sound velocity profile, Sound velocity profiles are usually measured over a certain time and space span.

Station layout of sound velocity profile involves three items: the number of stations in sound velocity profile, regional distribution and time distribution ${ }^{[4]}$. The basic principle of its layout is: on the premise of satisfying the accuracy requirement of multi-beam sound velocity correction, the measurement area control of sound velocity profile can be realized with the smallest sampling station ${ }^{[5]}$. In order to facilitate operation, the specific implementation requirements are given in the Technical Regulations for Multi-Beam Bathymetry ${ }^{[6]}$. Nevertheless, in practical work, the surveyors still feel difficult to grasp, and can not judge whether the required measurement can meet the accuracy requirements of multi-beam sound velocity correction in any sea area. In order to solve this problem, based on the constant gradient ray tracing algorithm, this paper constructs an acoustic profile accuracy evaluation model of the weakest point in water depth, evaluates the accuracy of the weakest point (the center point in time or space of sound velocity profile) caused by the difference of sound velocity profile, and takes the effective beam ratio as sound by setting the depth limit difference. According to the standard of velocity profile point layout, the reason of whether sound profile station should be installed is given, which can guide field surveyors to survey.

\section{Constant gradient layered sound tracking method}

The correction methods of sound velocity effect in multibeam sounding include the triangulation method, constant sound velocity layered ray tracing method, constant gradient sound velocity layered ray tracing method, relative area difference method and equivalent sound velocity profile method ${ }^{[7]}$. At present, constant gradient ray tracing method is commonly used and has the highest accuracy. In this paper, constant gradient ray tracing method is used in multi-beam sounding sound velocity correction. The constant gradient ray tracing method considers that the sound velocity in the water layer is a constant gradient change, and it is a method for 
calculating the position of the beam corner print based on the sampling of the sound velocity profile ${ }^{[8]}$. According to the stratification of adjacent sound velocity sampling points in the sound velocity profile, the change of sound velocity in the layer is considered as a constant gradient change. The constant gradient acoustic ray tracing method uses constant gradient layering to calculate the propagation time and horizontal displacement layer by layer. As shown in Figure 1, the accuracy of the constant gradient acoustic ray tracing method is the highest among various acoustic ray tracing methods, and it is also the most commonly used acoustic ray tracing method ${ }^{[9]}$.

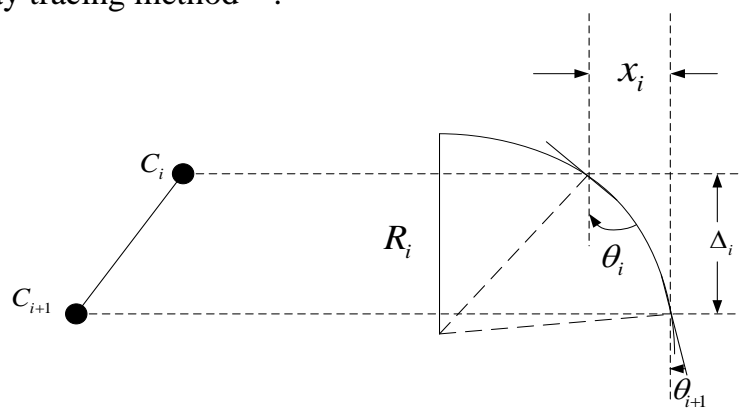

Fig. 1. Layered sketch of constant gradient ray tracing method The calculation formula is as follows:

$$
\begin{gathered}
P=\frac{\sin \theta_{0}}{C_{0}} \\
R_{i}=-\frac{1}{p g_{i}} \\
g_{i}=\frac{C_{i+1}-C_{i}}{\Delta i} \\
t i=\frac{R_{i}\left(\theta_{i}-\theta_{i+1}\right)}{C_{H i}}=\frac{\theta_{i+1}-\theta_{i}}{p g_{i}{ }^{2} \Delta_{i}} \operatorname{Ln}\left[\frac{C_{i+1}}{C_{i}}\right] \\
x_{i}=R_{i}\left(\cos \theta_{i+1}-\cos \theta_{i}\right)=\frac{\cos \theta_{i}-\cos \theta_{i+1}}{p g_{i}}
\end{gathered}
$$

Formulas for calculating stratification time and horizontal displacement with constant gradient can be obtained from formula (1) - (5):

$$
\begin{gathered}
t_{i}=\frac{a \sin \left[p\left(C_{i}+g_{i} \Delta_{i}\right)\right]-a \sin \left[p C_{i}\right]}{p g_{i}^{2} \Delta_{i}} \operatorname{Ln}\left[1+\frac{g_{i} \Delta_{i}}{C_{i}}\right] \\
x i=\frac{\sqrt{1-\left(p C_{i}\right)^{2}}-\sqrt{1-\left(p\left(C_{i}+g_{i} \Delta_{i}\right)\right)^{2}}}{p g_{i}}
\end{gathered}
$$

In the vertical direction, the layered time formula is as follows:

$$
t_{i}=\frac{1}{g} \operatorname{Ln} \frac{C_{i+1}}{C_{i}}
$$

Among them: $p$ means Snell constant; $\theta_{0}$ means beam angle of emission; $C_{0}$ means transducer surface sound velocity; $C_{i}$ means initial sound velocity of layer $i ; C_{i+1}$ means final sound velocity of layer $i ; \Delta_{i}$ means thickness of layer $i ; t_{i}$ means propagation time of sound wave in layer $i$; horizontal displacement of sound wave propagation in layer $i ; x_{i}$ means initial incident angle of layer $i ; \theta_{i}$ means initial refraction angle of layer $i$; $\theta_{i+1}$ means final refraction angle of layer $i ; g_{i}$ means sound velocity gradient in layer $i ; C_{H i}$ means the average sound velocity between layer $i$.

\section{Principle of weakness assessment}

In terms of time and space, there is a critical intermediate point in the control range of each station. This paper calls it the weakest point in the control range of acoustic dissection station ${ }^{[10]}$. The water depth measured at this point is called the weakest point in the control range of acoustic dissection station. By comparing the difference of water depth calculated by two acoustic profiles at this point, we can judge whether the layout of acoustic profiling stations is reasonable or not.

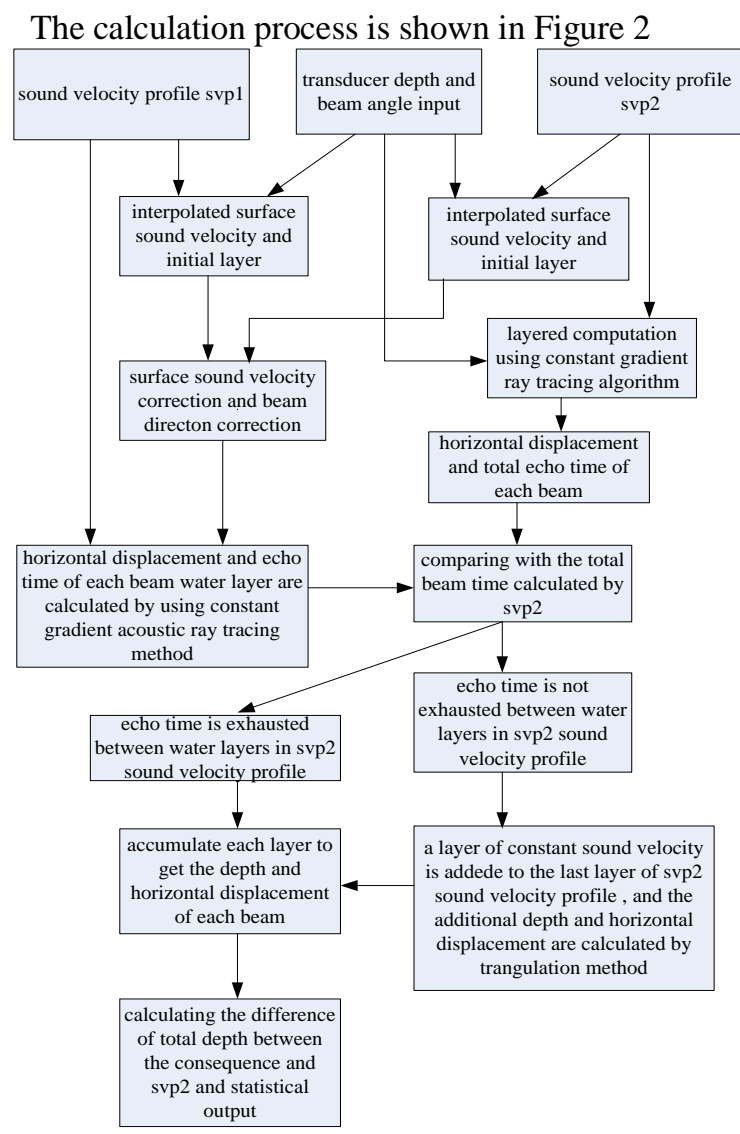

Fig. 2. Flow chart of sound velocity profile comparison Detailed steps are as follows:

(1) Read the sound velocity profile svp2 and initialize it.

(2) The calculation of echo time. The average transducer depth is $1 \mathrm{~m}$, and the surface acoustic velocity (SSV) of the transducer is interpolated as the initial sound velocity. The simulation chooses 1 unitary interval and 150 unitary beam coverage width as the initial transmitting angle of the beam. According to the constant gradient velocity tracking method, the echo time is obtained. At the same time, the depth of the current sound velocity profile is taken as the depth of the theoretical seabed. 
(3) Read the sound velocity profile svp1 and initialize it.

(4) Use svp1 and echo time to calculate the footprint position. If the echo time is exhausted in svp1, the final water depth is obtained by constant gradient ray tracing method; if the echo time exceeds the range of svp 1, the final water depth is calculated by triangulation method with the sound velocity of the last layer.

(5) Calculate the difference between the final calculated depth and the theoretical depth, that is, the correction difference caused by svp1 correction and svp2 correction. NOAA of the National Oceanic and Atmospheric Administration of the United States stipulated in 1999 that if the difference between known and actual sound velocity profiles for sounding correction exceeds $0.25 \%$ of the water depth, it will be deemed to exceed the limit ${ }^{[11]}$. In the calculation of this paper, $0.25 \% \mathrm{D}$ is chosen as the limit difference of water depth difference. The difference is compared with the limit difference, and the effective beam value is obtained. The effective beam ratio is the ratio of the effective wave value to the total beam. According to the regulation of NOAA, the control range of two acoustic profiles whose effective beam ratio is less than $80 \%$ can not be effectively connected, so it needs to be encrypted.

(6) Statistics and output results.

\section{Analysis of calculation example}

In May 2009, a survey unit made multi-beam measurements in the East China Sea. Three acoustic profiles were performed at 05:00, 11:28 and 21:50 on May 9, respectively. The acoustic profile data are shown in Figure 3.

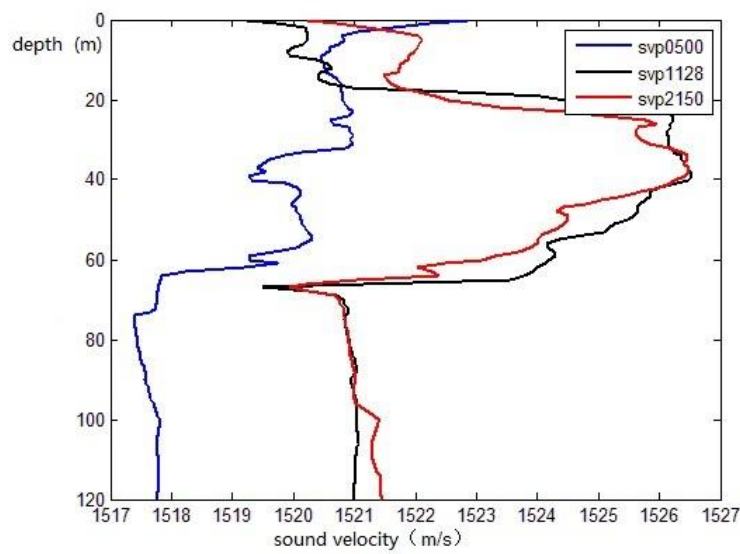

Fig. 3. Data distribution of different sound velocity profile

According to the above algorithm, the evaluation results of acoustic profile station control are shown in Table 1.

Table 1. Evaluation results of sound speed profile control station

\begin{tabular}{ccccc}
\hline $\begin{array}{c}\text { Comparing the } \\
\text { sound profile }\end{array}$ & $\begin{array}{c}\text { Station distance of } \\
\text { acoustic section }(\mathrm{km})\end{array}$ & $\begin{array}{c}\text { Range of mutual } \\
\text { difference }\end{array}$ & $\begin{array}{c}\text { Number of beams meet the } \\
\text { tolerance of } 0.25 \% \mathrm{~d}(\%)\end{array}$ & Effective \\
\hline svp0-svp1 & 57.97 & $-4.49 \sim 0.26$ & 69.54 & 52.2 \\
\hline svp0- svp2 & 58.33 & $-2.73 \sim 0.25$ & 77.48 & 58.1 \\
\hline svp1- svp2 & 2.59 & $0.0 \sim 1.44$ & 80.13 & 60.1 \\
\hline
\end{tabular}

From the data in the table, it can be seen that, according to the provisions of $0.25 \% \mathrm{~d}$ water depth difference limit difference and $80 \%$ effective beam ratio, only the density between svp1 and svp2 can meet the layout requirements on May 9, and that between svp0 and svp1, as well as between $\operatorname{svp} 0$ and svp2, can not meet the requirements of the weakest water depth effective beam ratio. The density of acoustic section station should be encrypted in space distance, otherwise the edge beam depth outside the effective beam width can not meet the requirements of sounding accuracy.

\section{Conclusion}

Sound velocity profile measurement is an indispensable part of multi-beam sounding, and its accuracy has a profound impact on the quality of water depth results ${ }^{[12]}$. In actual measurement, the sound velocity profile of discrete points is used to characterize the time-space variation of sound velocity in the whole measuring area. The density of acoustic section stations is the key factor to control the accuracy of sound velocity. Most of them are inefficient, but few of them can not meet the accuracy requirements. At present, most of the layout schemes are based on "Technical Regulations for MultiBeam Bathymetry"[13]. However, there is no corresponding evaluation method to determine the rigor of the scheme. Aiming at this problem, a model for evaluating the accuracy of acoustic profile based on constant gradient ray tracing algorithm is constructed. On the basis of evaluating the rationality of acoustical section station layout, the model provides adjustment advice for the following measurement tasks, optimizes the density of acoustical section station layout, and improves work efficiency on the premise of meeting the accuracy.

\section{References}

1. Zhang Zhiwei, Bao Jingyang, Jin Shan. An adaptive layering method for multi-beam sounding sound line tracking $[\mathrm{J}]$. Ocean Surveying and Mapping, 2018, 38(01): 23-26+42. 
2. Zheng Jian. Analysis of the influence of sound velocity on multi-Beam calibration accuracy[J]. Dam and Safety, 2017(06): 69-73.

3. Xiong Chuanliang, Li Jianjun, Yan Xincun, Liu Wei.Optimization of sampling interval for multibeam measurement of sound velocity rofile[J]. Ocean Surveying and Mapping,2017,37(04):47-50.

4. Huang Chenhu, Lu Xiuping, Ye Anna. Quality Check and Evaluation of Seabed Topographic Survey Results (2): Acquisition of Sound Velocity Profiles in Deep Sea Area [J]. Marine Surveying and Mapping, 2017, 37 (03): 12-16+20.

5. Lu Xiuping, Huang Motao, Zhai Guojun, Huang Xianyuan, Huang Chenhu. Progress and Prospect of Key Technologies in Multi-beam Sounding Data Processing [J]. Marine Surveying and Mapping, 2016, 36 (04): 1-6+11.

6. Du Guoyuan, Jinfa, Bi Junfang. The influence of sound velocity effect in precision sounding and the calculation of sound velocity correction [J]. Journal of Yangtze River Engineering Vocational and Technical College, 2016,33(01): 18-21+28.

7. Deng Zhijun, Huang Chenhu, Lu Xiuping, Qin Qingliang, Liu Min, Huang Xianyuan. Some problems in using multi-beam sounding sound velocity profile data $[\mathrm{J}]$. Marine Surveying and Mapping, 2015, 35 (05): 43-46.
8. Jin Shaohua, Sun Wenchuan, Wujingyang, Xiao Fumin, Cui Yang. Wedge representation of sound velocity errors in multi-beam bathymetry [J]. Marine Surveying and Mapping, 2015, 35 (03): 1115.

9. Sun Qiang, Li Mingsan, Wang Hongyan, Zhang Hao, Li Dongliang. Comparative analysis of sound ray tracking methods for multi-beam sounding systems [J]. Marine Surveying and Mapping, 2015, 35 (02): 48-51.

10. Sun Wenchuan, Storm Jingyang, Jin Shaohua. EOF Representation of Sound Velocity Profile on the Impact of Multi-Beam Bathymetric Data [J]. Marine Survey, 2014, 34 (06): 21-24+28.

11. Gao Jianqin, Zhao Kun. Dynamic Selection Method of Multi-beam Sound Velocity Profile [J]. Surveying and Mapping Engineering, 2014, 23 (11): 34-37.

12. Zhou Jian, Zhou Qing, Lu Liang, Chen Chun, Huanghai Ship. Discussion on Correction of Multibeam Sound Velocity Profile [J]. Marine Surveying and Mapping, 2014, 34 (04): 62-65+68.

13. Sun Qiang, Li Mingshan, Wang Hongyan, Xu Weiguo. Study on the determination method of acoustic profile density in sea-way survey [J]. Marine Survey and Mapping, 2013, 33 (06): 49-52. 